\title{
In vitro antimicrobial activity of tigecycline against Gram negative and Gram positive pathogens collected in Northen Italy (T.E.S.T. program 20I0)
}

Erika Coppo, Ramona Barbieri, Anna Marchese, Eugenio A. Debbia

Microbiology Section, D.I.S.C., University of Genoa, Genoa, Italy

Key words: Tigecycline, Broad-spectrum activity, Antibiotic-resistance

\section{SUMMARY}

Background. In this study (part of the global T.E.S.T. program) was evaluated the in vitro activity of tigecycline, member of a new class of antimicrobial agents, the glycylcyclines, against clinical isolates collected in Italy.

Methods. A total of 194 clinical isolates were collected and identified in our Institution during 20 I0. Minimum inhibitory concentrations (MICs) of the antimicrobial agents were determined by the CLSI (20I0) recommended broth microdilution method.

Results. Globally 129 Gram negative and 65 Gram positive pathogens were evaluated.Tigecycline demonstrated excellent inhibitory activity against Escherichia coli, Haemophylus influenzae, Enterococcus spp., Staphylococcus aureus MetS, Streptococcus pneumoniae and Streptococcus agalactiae with $M I C_{90} \leq 1 \mathrm{mg} / \mathrm{l}$.

Conclusion. Tigecycline exhibited potent in vitro antibacterial activity (comparable to or greater than most commonly employed antimicrobials) against both Gram positive and negative clinical pathogens. These data suggest that tigecycline, with an expanded broad-spectrum antimicrobial activity, may be an effective empiric therapeutic option for the treatment of serious infections caused by clinically relevant pathogens.

\section{INTRODUCTION}

Tigecycline is a first-in-class of expanded broadspectrum glycylcycline. It inhibits bacterial protein synthesis by binding to the 30 s ribosomial subunit, but with five times highter affinity than tetracyclines (2). This new drugs was approved for use in Europe in 2006 for complicated skin and soft-tissue and intra-abdominal infections (http://www.emea.europa.eu/humandocs/Humans /EPAR/tygacil/tygacil.htm).

In vitro studies demonstrate that it has good activity against many commonly encountered respiratory bacteria, including multiple resistant Gram positive, Gram negative, anaerobic, as well as multidrug-resistant (MDR) pathogens such methicillinresistant $S$. aureus (MRSA) and $S$. epidermidis (MRSE), vancomycin-resistant Enterococcus spp. (VRE), penicillin-resistant S. pneumoniae (PRSP) and $\beta$-lactamase producing $H$. influenzae (3).

Tygeciclyne inhibits bacterial protein biosynthesis blocking the attachment of amino-acyl tRNA to the A site of the ribosome and preventing the elongation of peptide chains (6).

The drug mantaines its activity even in presence of efflux pumps (encoded by tetA-tet $\mathrm{D}$ and tet $\mathrm{K}$ genes) and ribosomal protection (tet $\mathrm{M}$ ) mechanisms that otherwise confer tetracycline resistance. Tigecycline appears to overcame these mechanisms of resistance because of steric hin- drance due to the addition of a large substituent on the $\mathrm{D}$ ring at the 9 th position of the tetracycline molecule $(7,13)$.

This study is part of the larger global Tigecycline Evaluation and Surveillance Trials (T.E.S.T.) program and was undertaken to document the in vitro activity of tigecycline against clinical isolates collected in Northen Italy from in-patient and outpatient populations.

\section{MATERIALS AND METHODS}

A total of 194 clinical isolates were collected and identified to the species level in our Institution during 2010. Isolates were collected from both inpatients and outpatients with a documented infections in which the isolate collected was identified as the probable causative organism according to institutional criteria. Only one isolate per patient was accepted into the study.

Minimum inhibitory concentrations (MICs) of the antimicrobial agents were determined by the Clinical and Laboratory Standards Institute(CLSI) (11) recommended broth microdilution testing method. Overnight cultures of bacteria were diluted to give a final concentration of approximately $5 \times 10^{5}$ cells $/ \mathrm{ml}$. Samples were then added to equivalent volumes of the various concentration of antibiotics distributed on a microplate and prepared from serial twofold dilutions. After 18-24

\section{Corresponding author: Erika Coppo}

Sezione di Microbiologia , C.A. Romanzi, Facoltà di Medicina e Chirurgia - DISC

Largo Rosanna Benzi 10 - I6I32 Genova - Tel.: 0103538998 - Fax: 010353765 I

E-mail: erika.coppo@unige.it 
hours of incubation at $37^{\circ} \mathrm{C}$, the concentrations of drugs that prevented visible growth were recorded as the MICs. Tigecycline was supplied by Wyeth Pharmaceuticals (Collegeville, PA, USA). All other agents were supplied by the panel manufacturer, MicroScan (Dade Behring Inc., Sacramento, CA, USA).

The panel of agents tested against Gram positive organisms was: tigecycline (0.008-16), amoxicillin/clavulanic acid (0.03-8), ampicillin (0.06-16), ceftriaxone (0.03-64), meropenem (0.12-16), linezolid (0.5-8), levofloxacin (0.06-32), minocycline (0.25-8), penicillin (0.06-8), piperacillin/tazobactam (0.25-16) and vancomycin (0.12-32). The panel of antimicrobial agents tested against Gram negative organisms was: tigecycline (0.008-16), amikacin (0.5-64), amoxicillin/clavulanic acid (0.12-32), ampicillin (0.5-32), cefepime (0.5-32), ceftazidime (8-32), ceftriaxone (0.06-64), levofloxacin (0.06-32) meropenem (0.12-16), minocycline (0.25-8) piperacillin/tazobactam (0.06-128). MIC interpretive criteria established by CLSI (11) and recent US Food and Drug Administration guidelines for tigecycline (Tygacil, Product Insert. Wyeth Pharmaceuticals, Inc., Philadelphia, PA, USA, 2005) were followed. As no interpretative criterion for tigecycline with Acinetobacter baumannii has been established, Clinical and laboratory Standards Institute interpretative criteria for Enterobacteriaceae were used for A. baumannii, as had been reported in previous literature $(1,17)$. Quality controls (QC) were performed using the following strains: Escherichia coli ATCC 25922; E. coli ATCC 35218; H. influenzae ATCC 49766; $H$. influenzae ATCC 49247; S. aureus ATCC 29213; P. aeruginosa ATCC 27853; Enterococcus faecalis ATCC 29212 and S. pneumoniae ATCC 49619. Results were included in the analysis only when corresponding QC isolates tested within the acceptable range according to CLSI (2010).

\section{RESULTS}

The largest number of isolates was collected from in-patients population (medicine, surgery, ICU, nursing home rehabilitation, renal, infectious diseases, paediatrics and obstetrics), only $35 \%$ of strains derived from community acquired infections (Table 1).

Globally 129 Gram negative and 65 Gram positive pathogens were evaluated. The first group of bacteria included 25 E. coli, 25 Klebsiella spp., 25 Enterobacter spp., 10 Serratia marcescens, 20 P. aeruginosa, 15 A. baumannii, (80\% multi-drug resistant to cephalosporins aminoglycosides and quinolones simultaneously), $9 \mathrm{H}$. influenzae (11.1\% $\beta$-lactamase-producers). Gram positive strains included 15 Enterococcus spp. (6.6\% vancomycin-resistant), $25 S$. aureus (68\% methicillin-resistant), 15 S. pneumoniae (33.3\% penicillin-non-susceptible), $10 \mathrm{~S}$. agalactiae.

Bacterial isolates were obtained from blood (65, $33.5 \%)$, urine $(17,8.8 \%)$, respiratory tract $(53$, $27.3 \%)$, skin $(20,10.3 \%)$, wound $(13,6.7 \%)$, vagina swabs $(13,6.7 \%)$ and other site $(8,4.2 \%)$.

Table I. Distribution of the strains collected in this study according to different clinical settings.

\begin{tabular}{|c|c|c|c|c|c|c|c|c|c|c|c|c|}
\hline \multirow[t]{2}{*}{ Microorganism } & \multirow{2}{*}{ Tot } & \multirow{2}{*}{$\begin{array}{l}\text { Community } \\
\text { acquired }\end{array}$} & \multicolumn{10}{|c|}{ Hospital acquired } \\
\hline & & & Med & Sur & ICU & $\begin{array}{c}\text { Nursing } \\
\text { homel } \\
\text { rehab }\end{array}$ & Ren & ID & Ped & Obs & Unk & Other \\
\hline \multicolumn{13}{|l|}{ Gram negative } \\
\hline E. coli & 25 & 6 & 6 & 3 & 4 & 2 & & 1 & & & & 3 \\
\hline Klebsiella spp. (I) & 25 & 17 & 1 & 6 & & 1 & & & & & & \\
\hline Enterobacter spp. (2) & 25 & 4 & 5 & 6 & 4 & 1 & & 1 & & & & 4 \\
\hline S. marcescens & 10 & 1 & & & 5 & & & & & & I & 3 \\
\hline P. aeruginosa & 20 & 10 & 1 & & 5 & 1 & & & & & I & 2 \\
\hline A. baumannii & 15 & 5 & 3 & & 3 & 1 & & & & & & 3 \\
\hline H. influenzae & 9 & 2 & & & & & & 2 & 3 & & 1 & 1 \\
\hline \multicolumn{13}{|l|}{ Gram positive } \\
\hline S. agalactiae & 10 & 6 & & & & 1 & & & & 3 & & \\
\hline Enterococcus spp. (3) & 15 & 4 & & 1 & 4 & 1 & 1 & & 3 & & & 1 \\
\hline S. aureus & 25 & 9 & 4 & 1 & 2 & 3 & 1 & & & & 1 & 4 \\
\hline S. pneumoniae & 15 & 4 & & & 2 & & & & 8 & & 1 & \\
\hline
\end{tabular}

Med: medicine, Sur: surgery, ICU: Intensive Care Unit, NHIREH: Nursing homel rehabilitation, Ren: renal, ID: Infectious Desease, Ped: pediatrics, Obs: obstetrics, Unk: non-specified unit.

(I) K. pneumoniae 16, K. oxytoca 7; (2) E. cloacae 2 I, E. aerogenes 4; (3) E. faecalis 10, E. faecium 5. 
(Table 2).

Tigecycline demonstrate an excellent inhibitory activity against Enterobacteriaceae (Table 3), indeed tigecycline's $\mathrm{MIC}_{90}$ was minor or equal to 4 mg $\backslash$. Against E. coli and Klebsiella spp. only meropenem behaves better than tigecycline $\left(\mathrm{MIC}_{90} 0.12 \mathrm{mg} \backslash \mathrm{l}\right.$ and 0.25 $\mathrm{mg} \backslash 1$ respectively).

This new agent, as the other tetracyclines, showed limited in vitro activity against $P$. aeruginosa with $\mathrm{MIC}_{90}$ values equal to $16 \mathrm{mgll}$ (Table 4).

Tigecycline demonstrated a good inhibitory activity against A. baumannii $\left(\mathrm{MIC}_{50} 1 \mathrm{mg} \backslash \mathrm{l}\right.$ and $\mathrm{MIC}_{90} 2 \mathrm{mgll}$ ) even on multi-drug resistant strain, with the exception of a single isolate which showed a MIC value corresponding to $8 \mathrm{mg} \backslash \mathrm{l}$. Such result is consistent with recent reports of emergence of tigecycline-resistant $A$. baumannii after tigecycline therapy $(1,14)$ (Table 4). In vitro tigecycline's activity on $A$. baumannii was superior to beta-lactams, beta-lactams/beta-lactamase inhibitor combinations, cephalosporins, aminoglycosides and levofloxacin.

Tigecycline demonstrate excellent inhibitory activity against $H$. influenzae $\left(\mathrm{MIC}_{50}\right.$ and $\mathrm{MIC}_{90}$ $0.25 \mathrm{mg} \backslash \mathrm{l})$, included $\beta$-lactamase-producers strains (Table 4).

Against Gram positive isolates (Table 5), tigecycline shown an excellent activity against Enterococcus spp. The drug presented $\mathrm{MIC}_{90}$ of $0.25 \mathrm{mg} \backslash$, regardless of susceptibility to vancomycin. This value was the lowest of all comparative agents, in particular was several folds lower than linezolid, minocycline and levofloxacin.

The drug has shown a potent inhibitory activity against $S$. aureus regardless of methicillin-resistant phenotype. Tigecycline, with $\mathrm{MIC}_{50}$ and $\mathrm{MIC}_{90}$ values of 0.25 and $0.5 \mathrm{mg} / 1$, respectively, demonstrated in vitro activity comparable to vancomycin and greater than linezolid and levofloxacin against MRSA. In MSSA the $\mathrm{MIC}_{50}$ and $\mathrm{MIC}_{90}$ value was $0.25 \mathrm{mg} / 1$ and $0.5 \mathrm{mg} / 1$ respectively.

Tigecycline demonstrated potent inhibitory activity against $S$. agalactiae and $S$. pneumoniae (Table 6 ), even in penicillin-resistant strains, with $\mathrm{MIC}_{90}$ values $\leq 1 \mathrm{mg} \backslash$.

\section{DISCUSSION}

Resistance to currently available antibiotics and incidence of infection due to multidrug-resistant bacteria has dramatically increased worldwide during the last twenty years. In presence of a serious infection, appropriate empirical antibiotic therapy can be life-saving and the choice of an antimicrobial to which the pathogens are susceptible may be critical. For these reasons, the development of new antimicrobial agents with expanded antibacterial spectrum (increased activity against Gram negative as well as Gram positive microrganisms) is more urgent than ever $(5,18)$. Tigecycline's in vitro activity was comparable to or greater than most commonly prescribed antimicrobial against important clinical pathogens.

Tigecycline, for example, demonstrated on methicillin-resistant $S$. aureus (MRSA) in vitro activity comparable to vancomycin, the antimicrobial agents currently used for the treatment of serious staphylococcal infections and exhibits greater activity to linezolid against vancomycin-resistant Enterococcus faecalis and Enterococcus faecium (VRE). This promising compound may be usefull even in the treatment of serious infections caused by resistant Gram negative strains with limited therapeutic options. Tigecycline has shown to be highly effective agains E. coli and Klebsiella spp. A. baumannii is a problematic pathogen, particularly in ICUs. The results from TEST reveal that no antimicrobial agents tested was active against $A$. baumanni. Tigecycline was the only agent that shown a good inhibitory activity against $A$. baumannii that are commonly associated with serious nosocomial infections. Resistance of Acinetobacter to cephalosporins, aminoglycosides and quinolones is widespread with an 
increasing of multi-drug resistance $(9,8)$. Tigecycline is a potent antimicrobial agent even against the infections due to this important pathogen.

The drug demonstrated a limited activity, similar to other tetracyclines, only against $P$. aeruginosa.

The in vitro activity of tigecycline observed in this study suggests that this drug is a suitable antimicrobial agent for empiric treatment of serious infections sustained by some of the commonly encountered pathogens.

Tigecycline is available as parenteral agent, has linear pharmacokinetics, long terminal half-life and is extensively distributed in tissues (15).

Some interesting applications for this drug may be in surgical wound infections (particularly following abdominal surgery), and as alternative therapeutic agent in patient with serious allergies to $\beta$-lactam antibiotics (10).

Results from Phase III clinical studies in the treatment of complicated skin and skin structure infection (cSSSI) and complicated intra-abdominal infection (cIAI) have demonstrated the potential of tigecycline used as monotherapy for the treatment of this infections: the drug showed equivalence to imipenem in cIAI and to vancomycin plus aztreonam in cSSSI $(12,16,4,19)$.

The in vitro and in vivo studies points out that, tigecycline, with an expanded broad-spectrum antimicrobic activity against Gram positive and negative bacteria, offers a new alternative for the treatment of infections caused by clinically relevant pathogens in which the emergence of resistance to previously active antibiotics has created to the physicians limitations in therapeutic options.
Table 3. In vitro activity of tigecycline and comparative antimicrobial agents against Enterobacteriaceae clinical pathogens.

\begin{tabular}{|c|c|c|c|c|}
\hline \multirow[t]{2}{*}{ Microrganisms } & \multirow[t]{2}{*}{ Drugs } & MIC 50 & MIC 90 & MIC Range \\
\hline & & \multicolumn{3}{|c|}{$\mu g / L$} \\
\hline \multirow[t]{11}{*}{ E. coli } & Tigecycline & 0.5 & 1 & $0.06-8$ \\
\hline & Amikacin & 8 & 16 & $1-\geq 64$ \\
\hline & Amoxicillin Clavulanic Acid & 16 & 32 & $4-\geq 32$ \\
\hline & Ampicillin & $\geq 32$ & $\geq 32$ & $1-\geq 32$ \\
\hline & Cefepime & $\leq 0 ., 5$ & $\geq 32$ & $4-\geq 32$ \\
\hline & Ceftazidime & $\leq 8$ & $\geq 32$ & $\leq 8-\geq 32$ \\
\hline & Ceftriaxone & 0.25 & $\geq 64$ & $\leq 0.06-\geq 64$ \\
\hline & Levofloxacin & 8 & $\geq 8$ & $0.015-\geq 8$ \\
\hline & Meropenem & $\leq 0.06$ & 0.12 & $\leq 0.06-0.5$ \\
\hline & Minocycline & 8 & $\geq 16$ & $1 \geq 16$ \\
\hline & Piperacillin Tazobactam & $\geq 128$ & $\geq 128$ & $0.06-\geq 128$ \\
\hline \multirow[t]{11}{*}{ Klebsiella spp. } & Tigecycline & 0.5 & 2 & $0.25-2$ \\
\hline & Amikacin & 2 & 32 & $1-32$ \\
\hline & Amoxicillin Clavulanic Acid & 2 & 32 & $1-\geq 32$ \\
\hline & Ampicillin & $\geq 32$ & $\geq 32$ & $\geq 32$ \\
\hline & Cefepime & $\leq 0.05$ & $\geq 32$ & $\leq 0.05-\geq 32$ \\
\hline & Ceftazidime & $\leq 8$ & $\geq 32$ & $\leq 8-\geq 32$ \\
\hline & Ceftriaxone & $\leq 0.06$ & 64 & $\leq 0.06-\geq 64$ \\
\hline & Levofloxacin & 0.12 & $\geq 8$ & $0.03-\geq 8$ \\
\hline & Meropenem & $\leq 0.06$ & 0.25 & $\leq 0.06-1$ \\
\hline & Minocycline & 4 & 16 & $1-\geq 16$ \\
\hline & Piperacillin Tazobactam & 2 & 64 & $1-\geq 128$ \\
\hline \multirow[t]{11}{*}{ Enterobacter spp. } & Tigecycline & 1 & 4 & $0.5-\geq 16$ \\
\hline & Amikacin & 2 & 64 & $1-\geq 64$ \\
\hline & Amoxicillin Clavulanic Acid & $\geq 32$ & $\geq 32$ & $32-\geq 32$ \\
\hline & Ampicillin & $\geq 32$ & $\geq 32$ & $16-\geq 32$ \\
\hline & Cefepime & $\leq 0.5$ & $\geq 32$ & $\leq 0.5-\geq 32$ \\
\hline & Ceftazidime & $\leq 8$ & $\geq 32$ & $\leq 8-\geq 32$ \\
\hline & Ceftriaxone & 2 & $\geq 64$ & $0.12-\geq 32$ \\
\hline & Levofloxacin & 0.12 & 8 & $0.03-\geq 8$ \\
\hline & Meropenem & 0.25 & 8 & $\leq 0.06-8$ \\
\hline & Minocycline & 8 & $\geq 16$ & $2-\geq 16$ \\
\hline & Piperacillin Tazobactam & 4 & $\geq 128$ & $1-\geq 128$ \\
\hline \multirow[t]{11}{*}{ S. marcescens } & Tigecycline & 1 & 2 & $0.5-4$ \\
\hline & Amikacin & 2 & 8 & $1-16$ \\
\hline & Amoxicillin Clavulanic Acid & $\geq 32$ & $\geq 32$ & $8-\geq 32$ \\
\hline & Ampicillin & 32 & $\geq 32$ & $\leq 0,5-\geq 32$ \\
\hline & Cefepime & $\leq 0.5$ & $\leq 0,5$ & $\leq 0.5-1$ \\
\hline & Ceftazidime & $\leq 8$ & $\leq 8$ & $\leq 8$ \\
\hline & Ceftriaxone & 0.5 & 16 & $\leq 0.06-16$ \\
\hline & Levofloxacin & 1 & 4 & $0.25-8$ \\
\hline & Meropenem & 4 & $\geq 16$ & $0.06-\geq 16$ \\
\hline & Minocycline & 8 & 8 & $4-16$ \\
\hline & Piperacillin Tazobactam & 1 & 8 & $0.25-32$ \\
\hline
\end{tabular}


Table 4. In vitro activity of tigecycline and comparative antimicrobial agents against other Gram negative clinical pathogens.

\begin{tabular}{|c|c|c|c|c|}
\hline \multirow{2}{*}{ Microrganisms } & \multirow[t]{2}{*}{ Drugs } & MIC 50 & MIC 90 & MIC Range \\
\hline & & \multicolumn{3}{|c|}{$\mu g / L$} \\
\hline \multirow[t]{11}{*}{ A. baumannii } & Tigecycline & 1 & 2 & $0.25-8$ \\
\hline & Amikacin & $\geq 64$ & $\geq 64$ & $\leq 0.5-\geq 64$ \\
\hline & Amoxicillin Clavulanic Acid & $\geq 32$ & $\geq 32$ & $\leq 8-\geq 32$ \\
\hline & Ampicillin & $\geq 32$ & $\geq 32$ & $8-\geq 32$ \\
\hline & Cefepime & $\geq 32$ & $\geq 32$ & $\leq 0.06-\geq 32$ \\
\hline & Ceftazidime & $\geq 32$ & $\geq 32$ & $\leq 8-\geq 32$ \\
\hline & Ceftriaxone & $\geq 64$ & $\geq 64$ & $4-\geq 64$ \\
\hline & Levofloxacin & $\geq 8$ & $\geq 8$ & $0.06-\geq 8$ \\
\hline & Meropenem & $\geq 16$ & $\geq 16$ & $0.12-\geq 16$ \\
\hline & Minocycline & 16 & $\geq 16$ & $\leq 0.05-\geq 16$ \\
\hline & Piperacillin Tazobactam & $\geq 128$ & $\geq 128$ & $\leq 0.06-\geq 128$ \\
\hline \multirow[t]{11}{*}{$P$. aeruginosa } & Tigecycline & 8 & 16 & $8-\geq 16$ \\
\hline & Amikacin & 8 & $\geq 64$ & $2-\geq 64$ \\
\hline & Amoxicillin Clavulanic Acid & $\geq 32$ & $\geq 32$ & $\geq 32$ \\
\hline & Ampicillin & $\geq 32$ & $\geq 32$ & $\geq 32$ \\
\hline & Cefepime & 8 & $\geq 32$ & $2-\geq 32$ \\
\hline & Ceftazidime & 16 & $\geq 32$ & $\leq 8-\geq 32$ \\
\hline & Ceftriaxone & $\geq 64$ & $\geq 64$ & $64-\geq 64$ \\
\hline & Levofloxacin & 4 & $\geq 8$ & $0.25-\geq 8$ \\
\hline & Meropenem & 4 & 16 & $\leq 0.06-\geq 16$ \\
\hline & Minocycline & $\geq 16$ & $\geq 16$ & $4-\geq 16$ \\
\hline & Piperacillin Tazobactam & 16 & $\geq 128$ & $2-\geq 128$ \\
\hline \multirow[t]{11}{*}{ H. influenzae } & Tigecycline & 0.25 & 0.25 & $0.12-0.25$ \\
\hline & Amikacin & 2 & 4 & $2-8$ \\
\hline & Amoxicillin Clavulanic Acid & 1 & 2 & $0.25-2$ \\
\hline & Ampicillin & $\leq 0.5$ & 4 & $\leq 0.5-8$ \\
\hline & Cefepime & $\leq 0.5$ & $\leq 0.5$ & $\leq 0.5$ \\
\hline & Ceftazidime & $\leq 8$ & $\leq 8$ & $\leq 8-\geq 32$ \\
\hline & Ceftriaxone & $\leq 0.06$ & 0.5 & $\leq 0.06-16$ \\
\hline & Levofloxacin & 0.03 & 0.03 & $0.015-0.25$ \\
\hline & Meropenem & $\leq 0.06$ & 0.25 & $\leq 0.06-0.25$ \\
\hline & Minocycline & $\leq 0.5$ & I & $\leq 0.5-1$ \\
\hline & Piperacillin Tazobactam & $\leq 0.6$ & 0.25 & $\leq 0.06-8$ \\
\hline
\end{tabular}


Table 5. In vitro activity of tigecycline and comparative antimicrobial agents against Enterococcus spp. and Staphylococcus aureus.

\begin{tabular}{|c|c|c|c|c|}
\hline \multirow[t]{2}{*}{ Microrganisms } & \multirow[t]{2}{*}{ Drugs } & MIC 50 & MIC 90 & MIC Range \\
\hline & & \multicolumn{3}{|c|}{$\mu g / L$} \\
\hline \multirow[t]{11}{*}{ Enterococcus spp. } & Tigecycline & 0.12 & 0.25 & $0.03-0.5$ \\
\hline & Amoxicillin/Clavulanic Acid & 1 & $\geq 8$ & $0.5-\geq 8$ \\
\hline & Ampicillin & 2 & $\geq 16$ & $1-\geq 16$ \\
\hline & Ceftriaxone & $\geq 64$ & $\geq 64$ & $0.5-\geq 64$ \\
\hline & Levofloxacin & $\geq 32$ & $\geq 32$ & $0.5-\geq 32$ \\
\hline & Linezolid & 2 & 4 & $0.5-4$ \\
\hline & Meropenem & 8 & $\geq 16$ & $4-\geq 16$ \\
\hline & Minocycline & $\geq 8$ & $\geq 8$ & $\leq 0.25-\geq 8$ \\
\hline & Penicillin & 8 & $\geq 8$ & $2-\geq 8$ \\
\hline & Piperacillin/Tazobactam & 8 & $\geq 16$ & $4-\geq 16$ \\
\hline & Vancomycin & 1 & 4 & $1-\geq 32$ \\
\hline \multirow[t]{11}{*}{ S. aureus Met $R$} & Tigecycline & 0.25 & 0.5 & $0.12-16$ \\
\hline & Amoxicillin/Clavulanic Acid & 8 & $\geq 8$ & $0.12-\geq 8$ \\
\hline & Ampicillin & $\geq 16$ & $\geq 16$ & $\leq 0.06-\geq 16$ \\
\hline & Ceftriaxone & 64 & $\geq 64$ & $4-\geq 64$ \\
\hline & Levofloxacin & 16 & $\geq 32$ & $0.25-\geq 32$ \\
\hline & Linezolid & 4 & 4 & $\leq 0.5-4$ \\
\hline & Meropenem & 4 & $\geq 16$ & $\leq 0.12-\geq 16$ \\
\hline & Minocycline & 0.5 & $\geq 8$ & $\leq 0.25-\geq 8$ \\
\hline & Penicillin & $\geq 8$ & $\geq 8$ & $\leq 0.06-\geq 8$ \\
\hline & Piperacillin/Tazobactam & 8 & $\geq 16$ & $1-\geq 16$ \\
\hline & Vancomycin & 1 & 2 & $1-\geq 32$ \\
\hline \multirow[t]{11}{*}{ S. aureus Met S } & Tigecycline & 0.12 & 0.5 & $0.12-2$ \\
\hline & Amoxicillin/Clavulanic Acid & 4 & $\geq 8$ & $2-\geq 8$ \\
\hline & Ampicillin & $\geq 16$ & $\geq 16$ & $\geq 16$ \\
\hline & Ceftriaxone & 16 & $\geq 64$ & $4-\geq 64$ \\
\hline & Levofloxacin & 16 & 32 & $0.25-\geq 32$ \\
\hline & Linezolid & 8 & $\geq 8$ & $2-\geq 8$ \\
\hline & Meropenem & 1 & $\geq 16$ & $\leq 0.12-\geq 16$ \\
\hline & Minocycline & 0.5 & 2 & $\leq 0.25-4$ \\
\hline & Penicillin & $\geq 8$ & $\geq 8$ & $\geq 8$ \\
\hline & Piperacillin/Tazobactam & 8 & $\geq 16$ & $2-\geq 16$ \\
\hline & Vancomycin & 1 & 1 & 1 \\
\hline
\end{tabular}


Table 6. In vitro activity of tigecycline and comparative antimicrobial agents against Streptococci.

\begin{tabular}{|c|c|c|c|c|}
\hline \multirow[t]{2}{*}{ Microrganisms } & \multirow[t]{2}{*}{ Drugs } & MIC 50 & MIC 90 & MIC Range \\
\hline & & \multicolumn{3}{|c|}{$\mu g / L$} \\
\hline \multirow{11}{*}{$\begin{array}{l}\text { S. pneumoniae } \\
\text { PEN-R(5) }\end{array}$} & Tigecycline & 1 & 1 & $\mathrm{I}-2$ \\
\hline & Amoxicillin/Clavulanic Acid & 2 & 4 & $\mathrm{I}-4$ \\
\hline & Ampicillin & 4 & 4 & 4 \\
\hline & Ceftriaxone & 2 & 8 & $\mathrm{I}-8$ \\
\hline & Levofloxacin & 1 & 1 & I \\
\hline & Linezolid & 1 & 1 & 1 \\
\hline & Meropenem & 1 & 1 & $0.5-1$ \\
\hline & Minocycline & $\geq 8$ & $\geq 8$ & $\geq 8$ \\
\hline & Penicillin & 4 & 4 & $2-4$ \\
\hline & Piperacillin/Tazobactam & 4 & 4 & $4-4$ \\
\hline & Vancomycin & 0.5 & 1 & $0.5-1$ \\
\hline \multirow{11}{*}{$\begin{array}{l}\text { S. pneumoniae } \\
\text { PEN-S(I0) }\end{array}$} & Tigecycline & 0.25 & 0.5 & $0.25-1$ \\
\hline & Amoxicillin/Clavulanic Acid & $\leq 0.03$ & $\leq 0.03$ & $\leq 0.03-0.12$ \\
\hline & Ampicillin & $\leq 0.06$ & $\leq 0.06$ & $\leq 0.06-0.25$ \\
\hline & Ceftriaxone & $\leq 0.03$ & $\leq 0.03$ & $\leq 0.03-1$ \\
\hline & Levofloxacin & I & 1 & 1 \\
\hline & Linezolid & $\leq 0.5$ & 1 & $\leq 0.5-1$ \\
\hline & Meropenem & $\leq 0.12$ & $\leq 0.12$ & $\leq 0.12$ \\
\hline & Minocycline & 2 & 8 & $0.25-8$ \\
\hline & Penicillin & $\leq 0.06$ & $\leq 0.06$ & $\leq 0.06-0.25$ \\
\hline & Piperacillin/Tazobactam & $\leq 0.25$ & $\leq 0.25$ & $\leq 0.25$ \\
\hline & Vancomycin & 0.5 & 0.5 & $0.25-0.5$ \\
\hline \multirow[t]{11}{*}{ S. agalactiae } & Tigecycline & $\leq 0.03$ & 0.25 & $\leq 0.008-0.25$ \\
\hline & Amoxicillin/Clavulanic Acid & 0.06 & 0.06 & $\leq 0.03-0.06$ \\
\hline & Ampicillin & $\leq 0.06$ & $\leq 0.06$ & $\leq 0.06-0.12$ \\
\hline & Ceftriaxone & 0.06 & 0.06 & $\leq 0.03-1$ \\
\hline & Levofloxacin & 1 & 1 & $0.5-4$ \\
\hline & Linezolid & $\leq 0.5$ & 1 & $\leq 0.5-2$ \\
\hline & Meropenem & $\leq 0.12$ & $\leq 0.12$ & $\leq 0.12$ \\
\hline & Minocycline & 2 & $\geq 8$ & $\leq 0.25-\geq 8$ \\
\hline & Penicillin & $\leq 0.06$ & $\leq 0.06$ & $\leq 0.06$ \\
\hline & Piperacillin/Tazobactam & $\leq 0.25$ & $\leq 0.25$ & $\leq 0.25$ \\
\hline & Vancomycin & 2 & 0.5 & $\leq 0.12-0.5$ \\
\hline
\end{tabular}




\section{REFERENCES}

1. Anthony KB, et al. Clinical and microbiological outcomes of serious infections with multidrug-resistant Gram negative organisms treated with tigecycline. Clin Infect Dis 2008; 46: 567-70.

2. Bergeron J, Ammirati M, Danley D, et al. Glycylcyclines bind to the high-affinity tetracycline ribosomal binding site and evade $\operatorname{Tet}(\mathrm{M})$ - and $\operatorname{Tet}(\mathrm{O})$ mediated ribosomal protection. Antimicrob Agents Chemother 1996; 40: 2226-8.

3. Bhattacharya M, Parakh A, Narang M. Tigecycline. $J$ Postgrad Med 2009; 55: 65-8.

4. Breedt J, Teras J, Gardovskis J, et al. Tigecycline 305 cSSSI Study Group: Safety and efficacy of tigecycline in treatment of skin and skin structure infections: results of a double-blind phase 3 comparison study with vancomycin-aztreonam. Antimicrob Agents Chemother 2005; 49: 4658-66.

5. Bush K. Why it is important to continue antibacterial drug discovery. ASM News 2004; 70: 282-7.

6. Chopra I. Glycylcyclines: third-generation tetracycline antibiotics. Curr Opin Pharmacol 2001; 1: 464-9.

7. Fluit AC, Florijn A, Verhoef J, Milatovic D. Presence of tetracycline resistance determinants and susceptibility to tigecycline and minocycline. Antimicrob Agents Chemother 2005; 49: 1636-8.

8. Hanberger H, Garcia-Rodriguez JA, Gobernado M, Goossens H, Nilsson LE, Struelens MJ. Antibiotic susceptibility among aerobic Gram-negative bacilli in intensive care units in 5 European countries. French and Portuguese ICU Study Groups. Jama 1999; 281: 67-71.

9. Henwood CJ, Gatward T, Warner M, et al. Antibiotic resistance among clinical isolates of Acinetobacter in the UK, and in vitro evaluation of tigecycline (GAR936). J Antimicrob Chemother 2002; 49: 479-87.

10. Livermore DM. Tigecycline: what is it, and where should it be used? J Antimicrob Chemother 2005; 56: 611-4.

11. Methods for dilution antimicrobial susceptibility tests for bacteria that grow aerobically; approved standardeighth edition. CLSI document M07-A8. Clinical Laboratory Standards Institute, Wayne, Pennsylvania, 2010.

12. Oliva ME, Rekha A, Yellin A, et al. A multicenter trial of the efficacy and safety of tigecycline versus imipenem/cilastatin in patients with complicated intra-abdominal infections. BMC Infect Dis 2005; 5: 88.

13. Petersen PJ, Bradford PA, Weiss WJ, Murphy TM, Sum PE, Projan SJ. In vitro and in vivo activities of tigecycline (GAR-936), daptomycin, and comparative antimicrobial agents against glycopeptide-intermediate Staphylococcus aureus and other resistant Gram positive pathogens. Antimicrob Agents Chemother 2002; 46: 2595-601.

14. Reid GE, Grim SA, Aldeza CA, Janda WM, Clark NM. Rapid development of Acinetobacter baumannii resistance to tigecycline. Pharmacotherapy 2007; 27: 1198-201.

15. Rello J. Pharmacokinetics, pharmacodynamics, safety and tolerability of tigecycline. J Chemother 2005; 17 (S1): S12-S22.

16. Sacchidanand S, Penn RL, Embil JM, et al. Efficacy and safety of tigecycline monotherapy compared with vancomycin plus aztreonam in patients with complicated skin and skin structure infections: results from a phase 3, randomized, double-blind trial. Int $J$ Infect Dis 2005; 9: 251-61.

17. Schafer JJ, Goff DA, Stevenson KB, Mangino JE. Early experience with tigecycline for ventilator-associated pneumonia and bacteremia caused by multidrug-resistant Acinetobacter baumannii. Pharmacotherapy 2007; 27: 980-7.

18. Shlaes DM, Projan SJ, Edwards JE. Antibiotic discovery: state of the state. ASM News 2004; 70: 275-81.

19. Wilcox MH. Efficacy of tigecycline in complicated skin and skin structure infections and complicated intra-abdominal infections. J Chemother 2005; 17 (S1): S23-S29. 